\title{
Aldehyde dehydrogenase 2 (ALDH2) polymorphism gene and coronary artery disease risk: a meta-analysis
}

\author{
L.L. Zhang', Y.Q. Wang ${ }^{2}$, B. Fu' ${ }^{2}$, S.L. Zhao ${ }^{2}$ and Y. Kui ${ }^{3}$ \\ ${ }^{1}$ Department of Respiration Medicine, West China Hospital, \\ Sichuan University, Chengdu, China \\ ${ }^{2}$ Department of Cardiovascular Surgery, West China Hospital, \\ Sichuan University, Chengdu, China \\ ${ }^{3}$ Department of Orthopedics, Fifth People's Hospital in Chengdu, \\ Chengdu, China \\ Corresponding author: Y. Kui \\ E-mail: donglikn199@163.com
}

Genet. Mol. Res. 14 (4): 18503-18514 (2015)

Received August 8, 2015

Accepted October 14, 2015

Published December 23, 2015

DOI http://dx.doi.org/10.4238/2015.December.23.38

\begin{abstract}
We studied the association between aldehyde dehydrogenase 2 (ALDH2) polymorphism and coronary artery disease (CAD) and clarified the mechanisms underlying this association. We searched the ISI, Medline (Ovid), PubMed, CNKI, Wanfang, and Weipu Databases. Statistical analysis was performed using Revman 5.0 and Stata12.0 softwares. A total of 3305 cases and 5016 controls in 12 casecontrol studies were included in this meta-analysis. Variant A allele carriers showed a $48 \%$ increased risk of CAD compared with homozygote $A$ allele [odds ratio $(\mathrm{OR})=1.48,95 \%$ confidence interval $(\mathrm{CI})=1.18-1.87$ for $\mathrm{AA}$ $+\mathrm{AG} v \mathrm{sG}$. In subgroup analysis by gender, significantly elevated risks were found in the mixed group $(\mathrm{OR}=1.78,95 \% \mathrm{CI}=1.42-2.22)$ but not in males $(\mathrm{OR}=1.12,95 \% \mathrm{CI}=0.79-1.57)$. In subgroup analysis by disease type, significant elevated risks were associated with A allele carriers in myocardial infarction $[\mathrm{OR}=1.69,95 \% \mathrm{CI}=(1.05-2.71)]$, in coronary
\end{abstract}


heart disease $(\mathrm{OR}=1.36,95 \% \mathrm{CI}=1.00-1.86)$, but not in coronary heart disease plus diabetes mellitus subjects $(\mathrm{OR}=1.57,95 \% \mathrm{CI}=0.58-4.29)$. Moreover, those with the GG genotype consumed significantly more alcohol than those with the AA/AG genotypes (standard mean deviation: $6.32 \mathrm{~g}, 95 \% \mathrm{CI}=2.09-10.55, \mathrm{P}=0.000$ ). $\mathrm{ALDH} 2$ polymorphisms may be risk factors for CAD. Moreover, CAD patients with ALDH2 genotypes AG and AA consumed significantly less alcohol than those with GG. To further evaluate gene-gene and gene-environment interactions between ALDH2 polymorphisms and the risk of CAD, more studies with larger groups of patients are required.

Key words: Alcohol dehydrogenase 2; Coronary artery disease; Meta-analysis; Polymorphism

\section{INTRODUCTION}

Coronary artery disease (CAD) is a major cause of morbidity and mortality in humans worldwide. Mortality associated with ischemic heart disease has increased in recent years, and a better understanding of the pathophysiology of coronary atherosclerosis is critical for developing early intervention strategies. CAD, including myocardial infarction (MI) and coronary heart disease (CHD), is a complex disease caused by multiple genetic and environmental factors. Alcohol consumption is a major risk factor for the development of CAD. However, the association between alcohol consumption and CAD appears to be largely dependent on the amount consumed; excessive alcohol consumption appears to be associated with an increased CAD risk.

Alcohol is initially metabolized to an intermediate compound, acetaldehyde, which is further metabolized and then eliminated from the body. The major enzyme responsible for acetaldehyde elimination is alcohol dehydrogenase 2 (ALDH2). A sequence variant (rs671) on chromosome 12q24.2 was found to be associated with inactive ALDH2. A mutant allele, $A L D H 2 * 2$, contains a single point mutation ( $\mathrm{G}$ to A transition in exon 12) at position 1510 of the active $A L D H 2 * 1$ gene, which results in a substitution of glutamic acid 504 to lysine and inactivates the enzyme. The $A L D H 2 * 2$ single point mutation in ALDH2 is common in some Asian populations. Hence, 2 ALDH2 alleles exist with 3 genotypes, including * $1 / * 1 /$ GG (wild-type homozygote), *1/*2/AG (heterozygote), and *2/*2/AA (mutant homozygote). Individuals homozygous for the $A L D H 2 * 2$ allele have an 18 times higher peak blood acetaldehyde levels, while levels in heterozygotes are 5-fold higher compared with $* 1 * 1$ homozygotes (Amamoto et al., 2002). The ALDH2 enzyme is responsible for the detoxification of aldehydes generated by alcohol consumption and lipid peroxidation, including 4-hydroxynonenal (Wang et al., 2002). A genetic variant that decreases ALDH2 activity, the $A L D H 2$ polymorphism, is transmitted in an autosomal additive manner and is common in the Asian population. Wada et al. (2008) found no variants of rs671 in a Caucasian population.

Carriers of the $A L D H 2$ polymorphism were found to be at an increased risk of myocardial infarction and diabetes mellitus in Chinese patients with CAD (Xu et al., 2007). Additionally, serum concentrations of lipid peroxides were significantly higher in carriers of the rs671 A allele $(A L D H 2$ Lys504 or $A L D H 2 * 2)$ in Japanese women, even after correction for alcohol consumption (Ohsawa et al., 2003). Therefore, exploring the association between $A L D H 2$ polymorphisms and $\mathrm{CAD}$ is important for developing novel preventive and therapeu- 
tic strategies for treating CAD.

No previous meta-analysis study has assessed the association between this polymorphism and CAD. In this study, we examined whether the $A L D H 2$ rs671 polymorphism is associated with $\mathrm{CAD}$ in an Asian population. We conducted a comprehensive and systematic review including original studies using a meta-analysis approach with a focus on the relationship between $A L D H 2$ polymorphisms and CAD.

\section{MATERIAL AND METHODS}

\section{Publication search}

A literature search was conducted using electronic databases, including Medline (Ovid), PubMed, ISI, CNKI, WanFang, and Weipu, to determine the correlation between ALDH2 polymorphism and CAD risk (last search was updated on March 12, 2013). Terms related to "CAD risk" included (coronary artery disease or CAD) and (ALDH2 or alcohol dehydrogenase 2) and (polymorph* or mutation* or variant* or genotype*) as the key words were searched. Searching was performed in duplicate by 2 independent reviewers (L.L. Zhang and J. Gong). No language restrictions were used. Studies in our meta-analysis met the following inclusion criteria: i) the studies evaluated polymorphisms or/and CAD, ii) the studies were case-control studies, iii) genotype distributions were included for both cases and controls to estimate an odds ratio (OR) with $95 \%$ confidence interval $(95 \% \mathrm{CI})$, iv) genotype distributions in control population was consistent with Hardy-Weinberg equilibrium. Accordingly, the studies that were excluded were as follows: i) abstracts only and reviews, ii) repeat or overlapped publications, and iii) no data of genotype frequency.

\section{Data extraction}

Two reviewers independently checked all potentially relevant studies and reached a consensus on all items. In case of disagreement, a third reviewer evaluated the articles. The following data were collected from each study: first author, year of publication, ethnicity, definition of cases, the source of the control, the total number of cases and controls, and genotype distribution in cases and controls.

\section{Quality score assessment}

The quality of studies was also independently assessed by the same reviewers (G.W. Zhang and J. Gong) who used quality assessment scores modified from previous meta-analysis of molecular correlational studies (Thakkinstian et al., 2005). These scores included both traditional epidemiological considerations and genetic issues. Total scores ranged from 0 (worst) to 13 (best).

\section{Statistical analysis}

For each study, we first examined whether the genotype distribution in controls was in Hardy-Weinberg equilibrium by using an Internet-based program (http://www.changbioscience. $\mathrm{com} /$ genetics/hardy.html). The degree of $A L D H 2$ polymorphisms and CAD risk was measured by OR at the $95 \% \mathrm{CI}$. The statistical significance of OR was determined using a Z-test. The risk 
of $A L D H 2$ polymorphisms was first estimated using a recessive model (AA $v s \mathrm{GG}+\mathrm{AG}$ ) and dominant model (AA+AG vs GG), evaluated using the variant genotype AA, and compared with the wild-type GG homozygote using a codominant model. Additionally, the risks of A vs $\mathrm{G}$ and $\mathrm{AG}$ vs GG were estimated using an additive model.

Heterogeneity was evaluated by an $\chi^{2}$-based Q statistical analysis and statistical significance was considered at an $\mathrm{I}^{2}$ value of less than $50 \%$. When the $\mathrm{I}^{2}$ value was less than $50 \%$, the pooled OR of each study was calculated using the fixed-effect model; otherwise, a random-effect model was used. The significance of the pooled OR was determined using a Z-test, and statistical significance was considered to be $\mathrm{P}<0.05$. To evaluate disease type-specific and gender-specific effects, subgroup analysis was performed by disease type or gender group. Sensitivity analysis was performed by sequentially excluding individual studies to assess the stability of the results. Publication bias was analyzed by several methods: i) visual inspection of asymmetry in funnel plots and ii) Begg test and Egger test. All statistical tests were performed using the RevMan 5.0 software (www.cochrane.org) and Stata 12.0 (StataCorp., College Station, TX, USA).

\section{RESULTS}

\section{Study inclusion and characteristics}

The Medline (Ovid), PubMed, CNKI, Wanfang, and Weipu databases were comprehensively searched. There were 86 records relevant to our search strategy. After reading the titles and abstracts, 65 studies were excluded because they did not exmaine CAD risk and the $A L D H 2$ gene. The full-texts of the remaining 17 articles were reviewed (Takagi et al., 2002; Xue et al., 2007; Jo et al., 2007; Li, 2008; Wada et al., 2008; Chao, 2009; Yuan, 2009, 2010; Guo et al., 2010; Hao et al., 2010; Yijie, 2010; Yuguo, 2010; Xu et al., 2007, 2010, 2011; Kotani et al., 2012; Ping, 2012). Next, an additional 6 articles were excluded [2 were repeated or overlapping studies (Li, 2008; Yuan, 2009), 2 were not case-control studies (Ji, 2008; Li, 2012); and 2 did not include the full-text (Wada et al., 2008; Kotani et al., 2012)]. A total of 12 articles were left for data extraction. One article reported 2 cohorts, and each cohort was considered as a separate case-control study. Thus, a total of 13 case-control studies were identified. Additionally, the genotypes in controls for 4 case-control studies were not consistent with Hardy-Weinberg equilibrium, and these studies were excluded (Kong et al., 2012). Finally, a total of 11 case-control studies in 11 articles met our inclusion criteria (Takagi et al., 2002; Xue et al., 2007; Jo et al., 2007; Chao, 2009; Guo et al., 2010; Bian et al., 2010; Yuguo, 2010; Xu et al., 2007, 2010, 2011; Ping, 2012), including 3305 cases and 5061 controls. The characteristics of each case-control are listed in Table 1. There were 5 cases-controls of MI (Takagi et al., 2002; Xue, 2007; Jo et al., 2007; Li, 2008; Bian et al., 2010), 4 of CHD (Xu et al. 2007; Guo et al., 2010; Yuguo, 2010; Ping, 2012), 2 of CHD plus diabetes mellitus (Chao, 2009; Xu et al., 2010), and 1 of acute coronary syndrome (Xu et al., 2011). Four studies included unpublished material (Chao, 2009; Yuan, 2009; Yijie, 2010; Ping, 2012).

All studies were population-based and most participants were Japanese, Chinese, and Korean. Tables 2 and 3 show the associations between genotype and alcohol intake, and between genotype and potential confounders, with $\mathrm{P}$ values taken from the original papers. All studies showed substantial differences in alcohol intake by genotype among men or different type of CHD (included MI and CHD plus diabetes mellitus); among women, alcohol intake was very low, but the studies showed the same trends as in men. 


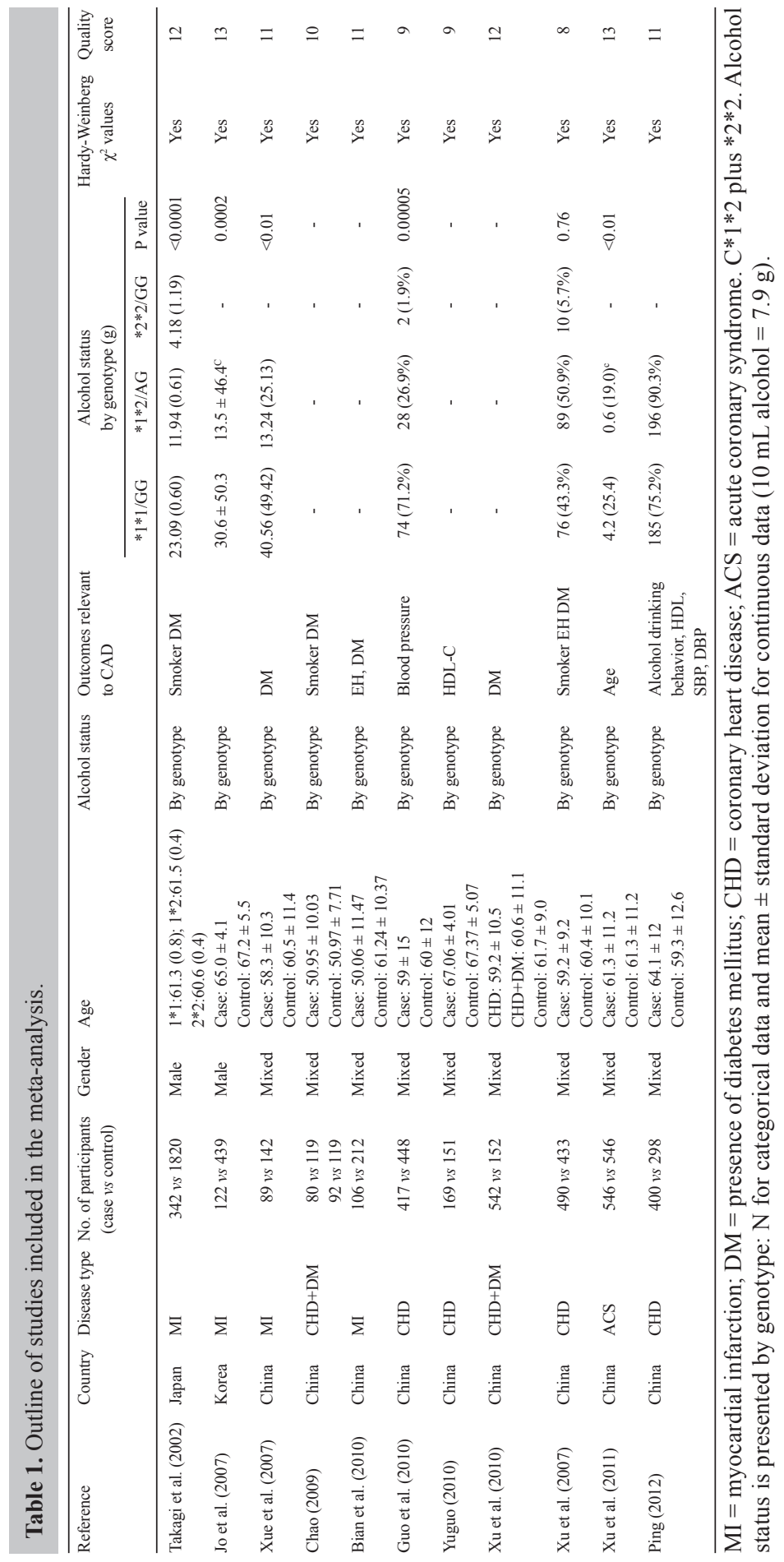


Table 2. Distribution of potential confounding factors by genotype among studies included in the meta-analysis.

\begin{tabular}{|c|c|c|c|c|c|c|c|}
\hline \multirow[t]{2}{*}{ Author } & \multirow[t]{2}{*}{ Category } & \multirow{2}{*}{\multicolumn{2}{|c|}{ Covariates }} & \multicolumn{3}{|c|}{ Covariates by genotypes ${ }^{\mathrm{a}}$} & \multirow{2}{*}{$\begin{array}{l}\text { Published } \\
\text { P values }\end{array}$} \\
\hline & & & & $* 1 * 1$ & $* 1 * 2$ & $* 2 * 2$ & \\
\hline \multirow[t]{3}{*}{ Takagi et al. (2002) } & Male & \multicolumn{2}{|c|}{ Age } & $60.6(0.4) 0$ & $61.5(0.4)$ & $61.3(0.8)$ & NS \\
\hline & & \multicolumn{2}{|c|}{$\%$ smoker } & 47.7 & 47.9 & 48.5 & - \\
\hline & & \multicolumn{2}{|c|}{ \%DM } & 40.6 & 37.7 & 23.5 & - \\
\hline \multirow[t]{3}{*}{ Jo et al. (2007) } & Male & \multicolumn{2}{|c|}{ Drinkers } & $30(24.0 \%)$ & $95(76.0 \%)$ & - & $<0.0001$ \\
\hline & & \multicolumn{2}{|c|}{ Ever drinkers } & $345(79.1 \%)$ & $91(20.9 \%)$ & - & \\
\hline & & \multicolumn{2}{|c|}{ Alcohol consumption (g/day) } & $30.6 \pm 50.3$ & $13.5 \pm 46.4$ & - & 0.0002 \\
\hline \multirow[t]{2}{*}{ Xue et al. (2007) } & Mixed & \multicolumn{2}{|c|}{ EH } & $90(61.2 \%)$ & $48(55.8 \%)$ & - & NS \\
\hline & & \multicolumn{2}{|c|}{ HDL-C } & $1.19 \pm 0.32$ & $1.18 \pm 0.29$ & - & NS \\
\hline \multirow{2}{*}{ Chao (2009) } & Mixed & \multicolumn{2}{|c|}{ SBP } & $126.08 \pm 16.13$ & $126.04 \pm 15.07$ & - & 0.032 \\
\hline & & \multicolumn{2}{|l|}{ BDP } & $77.28 \pm 8.69$ & $76.25 \pm 9.66$ & - & 0.046 \\
\hline Bian et al. (2010) & - & \multicolumn{2}{|l|}{ - } & - & - & - & - \\
\hline Guo et al. (2010) & Mixed & \multicolumn{2}{|c|}{ HDL-C } & $1.39 \pm 0.36$ & - & $1.02 \pm 0.02$ & 0.015 \\
\hline Yuguo $(2010)$ & Mixed & \multicolumn{2}{|l|}{-} & - & - & - & - \\
\hline \multirow[t]{6}{*}{$\mathrm{Xu}$ et al. (2010) } & & \multicolumn{2}{|c|}{ Drinking frequency } & 101 & 50 & - & - \\
\hline & & \multirow[t]{2}{*}{ Femal } & $<1$ drink & 0 & 0 & - & \\
\hline & & & $>5$ drinks & 0 & 0 & - & - \\
\hline & & \multirow[t]{3}{*}{ Male } & $<1$ drink & 35 & 29 & - & - \\
\hline & & & 1-4 drinks & 42 & 16 & - & - \\
\hline & & & $>5$ drinks & 29 & 6 & - & - \\
\hline \multirow[t]{4}{*}{ Xu et al. (2007) } & Mixed & \multicolumn{2}{|l|}{ EH } & $139(57.4 \%)$ & $86(35.5 \%)$ & $17(7 \%)$ & 0.72 \\
\hline & & \multicolumn{2}{|l|}{ DM } & $37(68.8 \%)$ & $20(34.5 \%)$ & $1(1.7 \%)$ & 0.73 \\
\hline & & \multicolumn{2}{|l|}{ HDL } & $127 \pm 51.2$ & $88 \pm 38.6$ & $19 \pm 8.3$ & 0.09 \\
\hline & & \multicolumn{2}{|c|}{ Smoker } & $148(53.2 \%)$ & $118(42.2 \%)$ & $12(4.4 \%)$ & 0.72 \\
\hline Xu et al. (2011) & Mixed & $\begin{array}{l}\text { Freque } \\
(>1 \text { day }\end{array}$ & $\begin{array}{l}\text { y of drinking } \\
\text { per week) }\end{array}$ & $31(49.2 \%)$ & $17(28.8 \%)$ & - & 0.034 \\
\hline Ping (2012) & Mixed & HDL & & $1.26 \pm 0.32$ & $1.24 \pm 0.35$ & - & 0.695 \\
\hline & & SBP & & $147.4 \pm 33.3$ & $145.9 \pm 34.3$ & - & 0.791 \\
\hline & & DBP & & $87.0 \pm 16.2$ & $87.1 \pm 17.4$ & - & 0.956 \\
\hline
\end{tabular}

$\mathrm{SBP}=$ systolic blood pressure; $\mathrm{DBP}=$ diastolic blood pressure; $\mathrm{EH}=$ essential hypertension; $\mathrm{DM}=$ presence of diabetes mellitus. ${ }^{a}$ Covariant status is presented by genotype: $\mathrm{N}(\%)$ for categorical data and mean standard deviation for continuous data. ${ }^{b} \mathrm{P}$ values were according to the original report from papers. $\mathrm{NS}=$ reported the result as not significant.

Table 3. Distribution of ALDH2 genotype among patients with CAD and controls included in the meta-analysis.

\begin{tabular}{|c|c|c|c|c|c|c|c|c|c|c|c|}
\hline \multirow[t]{2}{*}{ Author } & \multirow[t]{2}{*}{ Year } & \multicolumn{3}{|c|}{ CHD } & \multicolumn{2}{|c|}{ Allele } & \multicolumn{3}{|c|}{ Control } & \multicolumn{2}{|c|}{ Allele } \\
\hline & & GG & GA & $\mathrm{AA}$ & G & A & GG & GA & AA & G & A \\
\hline Takagi & 2002 & 160 & 139 & 43 & 459 & 225 & 875 & 786 & 159 & 2536 & 1104 \\
\hline Jo & 2007 & 70 & 48 & 4 & 188 & 56 & 305 & 122 & 12 & 732 & 146 \\
\hline Xue & 2007 & 39 & 47 & 3 & 125 & 53 & 106 & 35 & 1 & 247 & 37 \\
\hline $\mathrm{Xu}$ & 2007 & 59 & 42 & 0 & 160 & 42 & 17 & 13 & 0 & 47 & 13 \\
\hline Chao & 2009 & 39 & 43 & 10 & 121 & 63 & 79 & 34 & 6 & 192 & 46 \\
\hline Bian & 2010 & 54 & 48 & 4 & 156 & 56 & 146 & 63 & 3 & 355 & 69 \\
\hline Guo & 2010 & 219 & 171 & 27 & 609 & 225 & 291 & 135 & 22 & 717 & 179 \\
\hline Chao & 2009 & 92 & 53 & 24 & 237 & 101 & 151 & 47 & 11 & 349 & 69 \\
\hline $\mathrm{Xu}$ & $2010^{\mathrm{a}}$ & 131 & 111 & 15 & 373 & 141 & 101 & 95 & 6 & 297 & 107 \\
\hline $\mathrm{Xu}$ & $2010^{\mathrm{b}}$ & 177 & 100 & 8 & 454 & 116 & 101 & 95 & 6 & 297 & 107 \\
\hline $\mathrm{Xu}$ & 2011 & 253 & 202 & 34 & 708 & 270 & 240 & 173 & 20 & 653 & 213 \\
\hline $\mathrm{Xu}$ & 2011 & 291 & 245 & 1 & 827 & 247 & 372 & 173 & 1 & 917 & 175 \\
\hline Ping & 2012 & 192 & 208 & 0 & 592 & 208 & 161 & 128 & 0 & 450 & 128 \\
\hline
\end{tabular}

${ }^{a, b}$ From the same study, but different groups, ${ }^{a} \mathrm{CHD}+\mathrm{DM}$ group, ${ }^{\mathrm{b}}$ only $\mathrm{CHD}$ group.

Meta-analysis with CAD as the outcome included 8366 participants. As shown in Figure 1, we analyzed the heterogeneity of AA + AG vs GG for all 11 studies and the value 
of $\mathrm{I}^{2}$ was $81 \%$ and Phet $=0.101$, suggesting very large heterogeneity. Thus, we chose the randomized-effects model to synthesize the data. The overall $\mathrm{OR}$ was $1.48(95 \% \mathrm{CI}=1.18$ $1.87)$ and the test for overall effect showed a $\mathrm{Z}$ value of $4.06(\mathrm{P}=0.001)$. These results suggest that A allele carriers are at a $48 \%$ increased risk of CAD compared with individuals with the GG homozygote. A summary of the results of other genetic comparisons is listed in Table 4.

Study
ID

Figure 1. Meta-analysis with a random-effect model for the association between CAD risk and the ALDH2 polymorphism (AA +AG vs GG).

Subgroup analyses were performed after stratification of the data by disease type and gender. In subgroup analysis, increased risks were identified among $\mathrm{MI}(\mathrm{OR}=1.69,95 \% \mathrm{CI}$ $=1.05-2.71) ; \mathrm{I}^{2}=82.1 \%$ for heterogeneity was observed for the dominant model AA $+\mathrm{AG} v s$ $\mathrm{GG}$ (Figure 2$)$ and in $\mathrm{CHD}(\mathrm{OR}=1.36,95 \% \mathrm{CI}=1.00-1.86)$, but not in CHD plus DM subjects $(\mathrm{OR}=1.57,95 \% \mathrm{CI}=0.58-4.29) . \mathrm{I}^{2}=88.8 \%$ for heterogeneity with a dominant model AA + AG vs GG. Thus, MI carriers of the A allele showed an increased risk of CAD. In subgroup analysis by gender, significantly increased risks were identified among mixed subjects $(\mathrm{OR}=$ $1.78,95 \% \mathrm{CI}=1.42-2.22), \mathrm{I}^{2}=82.2 \%$ for heterogeneity and the $\mathrm{Z}$ test for overall effect was $3.12(\mathrm{P}=0.071)$. However, among males, there was no significant association with $\mathrm{CAD}$ risk in the recessive model $(\mathrm{OR}=1.12,95 \% \mathrm{CI}=0.79-1.57)$ and the $\mathrm{Z}$ test for overall effect was $1.09(\mathrm{P}=0.063$; Figure 3$)$. 


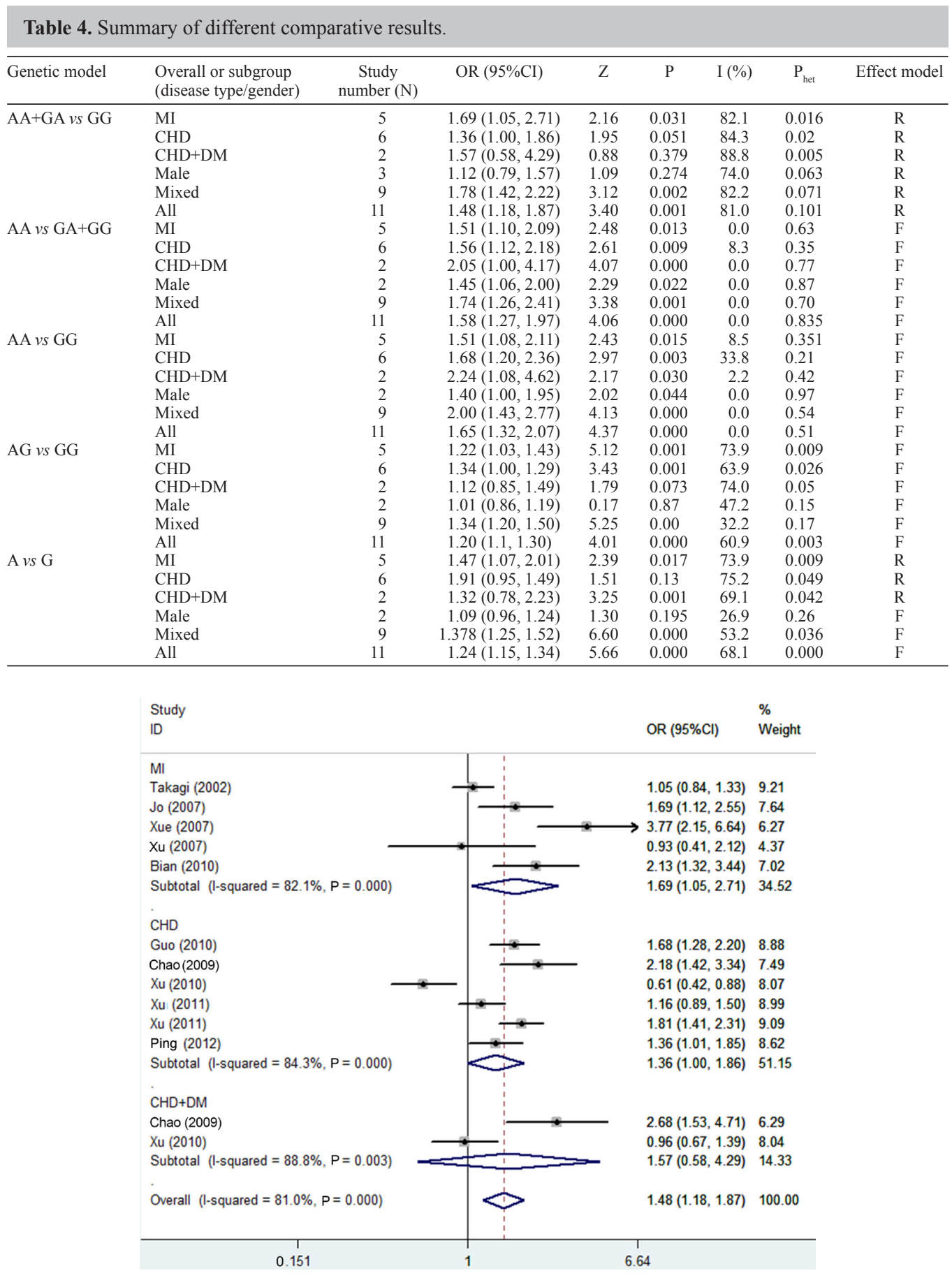

Figure 2. Meta-analysis with a random-effect model for the association between CAD risk and the ALDH2 polymorphism (AA +AG vs GG), subgroup analysis by disease type. 


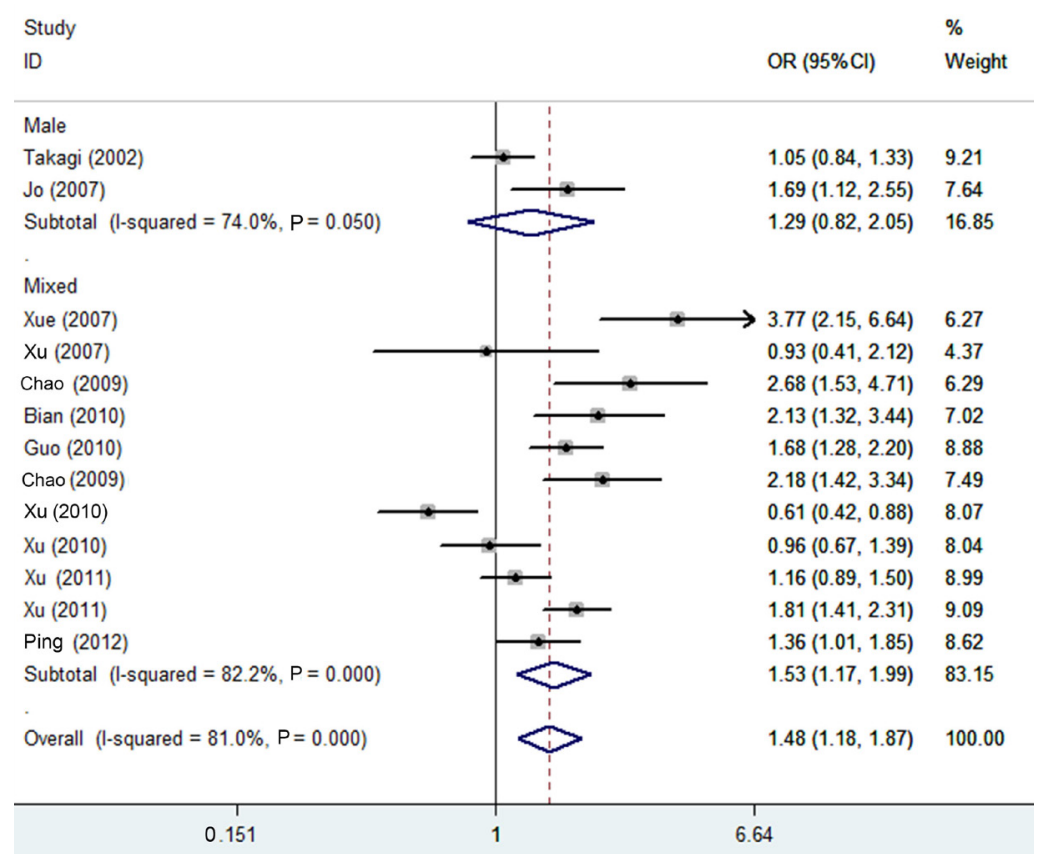

Figure 3. Meta-analysis with a random-effect model for the association between CAD risk and the ALDH2 polymorphism (AA+AG vs GG), subgroup analysis by gender.

We further showed that patients with $A L D H 2$ genotype GG consumed more alcohol than those with the $\mathrm{AA} / \mathrm{AG}$ genotypes (standard mean deviation: $6.32 \mathrm{~g}, 95 \% \mathrm{CI}=2.09-10.55$, $\mathrm{P}=0.000$; Figure 4).

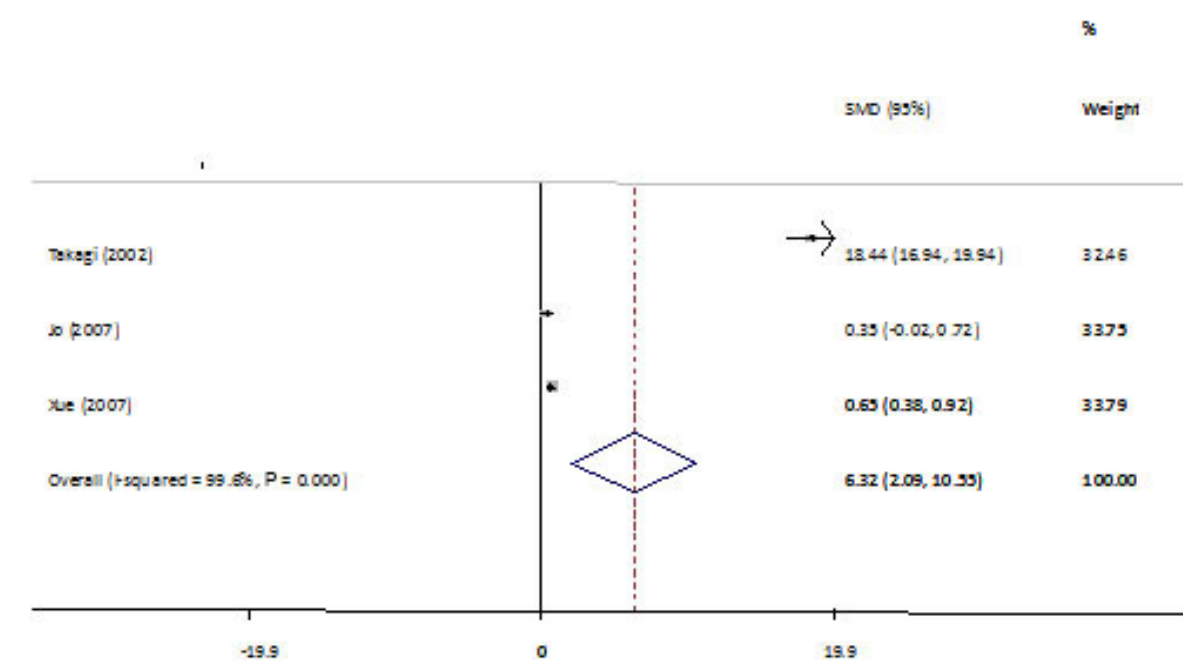

Figure 4. Meta-analysis with a random-effect model for the alcohol consumption between GG genotypes and AG+AA genotypes. 


\section{DISCUSSION}

We carried out the first meta-analysis to examine the association between the ALDH2 genotype and CAD. In addition, the risk of CAD among those with the A allele was 1.58 -fold above that among GG homozygotes. We further showed that CAD patients with the $A L D H 2$ GG genotype consumed significantly more alcohol than those with AG and AA.

Previous studies demonstrated that the frequency of $A L D H 2$ A allele differed significantly among different races (Thomasson et al., 1991; Muramatsu et al., 1995). The ALDH2 A allele mainly exists in East Asians (30-50\%), and approximately $6 \%$ of the world's population carries an $A L D H 2 \mathrm{G}$ allele. The distribution of $A L D H 2 \mathrm{~A}$ alleles also differs among different East Asian populations (Takagi et al., 2002; Carlsson et al., 2003). Previous studies showed that the $A L D H 2$ genetic polymorphism plays an important role in several pathological conditions, including hepatitis and certain types of carcinomas (Nomura et al., 2000; Yokoyama et al., 2003).

Recent studies suggested that this polymorphism is also associated with CAD. Our study confirmed this conclusion. The mechanisms underlying this association have not been fully clarified, but may be explained based on the following reasons. First, the A allele was previously reported to be associated with lower serum high-density lipoprotein-C levels independently of alcohol consumption in Japanese subjects (Wada et al., 2008), so the ALDH2 rs671 polymorphism may influence the risk of CAD at least in part through its effects on serum high-density lipoprotein-C levels. Moreover, the $A L D H 2$ rs671 polymorphism may increase the risk of CAD by increasing intracellular asymmetric dimethylarginine levels (Guo et al., 2010). Third, moderate consumption of alcohol has been consistently associated with a reduced risk of MI (Gaziano et al., 1993; Hines et al., 2001). Heavier alcohol consumption, in contrast, is associated with no change or even an increase in this risk (Mukamal et al., 2001). In our study, the $A L D H 2$ genetic polymorphism was also significantly associated with alcohol consumption and influenced the risk of CAD. Fourth, $A L D H 2$ genetic polymorphism may increase blood pressure, influencing the risk of CAD (Yamada et al., 2002).

It should be noted that the significant association between the $A L D H 2$ gene polymorphism and CAD in our study was observed based on a dominant model of inheritance. We observed an effect of the $A L D H 2$ A allele in all models used in this meta-analysis, but in Japanese and Korean studies we did not reach the same conclusion (Takagi et al., 2002; Jo et al., 2007). Although the reasons for these conflicting results remain unknown, they may be attributable to the significant differences in the frequencies of $A L D H 2$ genotypes between the Japanese and Korean populations.

There were some limitations to our study. First, some studies were excluded because the original genotype number or frequencies were not reported, which may have led to selection bias. Additionally, in some studies, the original data could not be obtained. Thus, these studies were excluded. Second, in this study, all eligible studies were published in English and Chinese from selected databases. It is possible that some relevant studies published in other languages were missed. Third, we should analyze the possibility of publication bias. Publication bias can result in the disappearance of some studies with negative results. Fourth, most of the studies were conducted in Asian subjects; thus, our study may only be applicable to Asians.

In conclusion, we found that the A allele of $A L D H 2$ mutant genotypes was a risk factor for CAD and that CAD patients with $A L D H 2$ genotypes AG and AA consumed significantly less alcohol than those with GG. These findings are valuable for understanding the pathogenic link between CAD in East Asians, which may lead to more effective CAD management. 
Moreover, the importance of the $A L D H 2$ polymorphism must be further investigated in prospective studies including different populations.

\section{Conflicts of interest}

The authors declare no conflict of interest.

\section{ACKNOWLEDGMENTS}

We followed PRISMA statement, and we thank Jin Gong and Gongwei Zhang for assistance with the meta analyses, data analysis, and figure preparation.

\section{REFERENCES}

Amamoto K, Okamura T, Tamaki S, Kita Y, et al. (2002). Epidemiologic study of the association of low-Km mitochondrial acetaldehyde dehydrogenase genotypes with blood pressure level and the prevalence of hypertension in a general population. Hypertens. Res. 25: 857-864.

Bian Y, Chen YG, Xu F, Xue L, et al. (2010). The polymorphism in aldehyde dehydrogenase-2 gene is associated with elevated plasma levels of high-sensitivity C-reactive protein in the early phase of myocardial infarction. Tohoku J. Exp. Med. 221: 107-112.

Carlsson S, Hammar N, Grill V and Kaprio J (2003). Alcohol consumption and the incidence of type 2 diabetes: a 20-year follow-up of the Finnish twin cohort study. Diabetes Care 26: 2785-2790.

Chao KC (2009). The Association of Aldehyde Dehydrogenase-2 Polymorphisms with Coronary Heart Disease in Type 2 Gene Diabetics. Shangdong University, Shandong.

Gaziano JM, Buring JE, Breslow JL, Goldhaber SZ, et al. (1993). Moderate alcohol intake, increased levels of high-density lipoprotein and its subfractions, and decreased risk of myocardial infarction. N. Engl. J. Med. 329: 1829-1834.

Guo YJ, Chen L, Bai YP, Li L, et al. (2010). The ALDH2 Glu504Lys polymorphism is associated with coronary artery disease in Han Chinese: relation with endothelial ADMA levels. Atherosclerosis 211: 545-550.

Hao PP, Xue L, Wang XL, Chen YG, et al. (2010). Association between aldehyde dehydrogenase 2 genetic polymorphism and serum lipids or lipoproteins: a meta-analysis of seven East Asian populations. Atherosclerosis 212: 213-216.

Hines LM, Stampfer MJ, Ma J, Gaziano JM, et al. (2001). Genetic variation in alcohol dehydrogenase and the beneficial effect of moderate alcohol consumption on myocardial infarction. N. Engl. J. Med. 344: 549-555.

Ji Z (2008). In vivo study on the relationship between ALDH2 gene polymorphism and efficacy of nitroglycerin in patient with stable angina. Master's thesis, Fudan University Department of Internal Medicine, Shanghai.

Jo SA, Kim EK, Park MH, Han C, et al. (2007). A Glu487Lys polymorphism in the gene for mitochondrial aldehyde dehydrogenase 2 is associated with myocardial infarction in elderly Korean men. Clin. Chim. Acta 382: 43-47.

Kong YQ, Wang ZX and Yun ML (2012). Correlation of rs671 polymorphism and acute myocardial infarction in the youth of Hainan Han nationality. China Trop. Med. 459: 60-65.

Kotani K, Sakane N and Yamada T (2012). Associatoin of an aldehyde dehydrogenase 2 (ALDH2) gene polymorphism with hyper-low-density lipoprotein cholesterolemia in a Japanese population. Ethn. Dis. 22: 324-328.

Li X (2008). The relationship between the levels of circulating endothelial progenitor cells and Aldehyde dehydrogenase-2 polymorphisms in patients with acute myocardial infarction. Master's thesis, Shangdong University, Shangdong.

Li X (2012). Role of aldehyde dehydrogenase 2 in the effects of ethanol on endothelial protection. Shangdong University, Shandong.

Mukamal KJ, Jadhav PP, D'Agostino RB, Massaro JM, et al. (2001). Alcohol consumption and hemostatic factors: analysis of the Framingham Offspring Cohort. Circulation 104: 1367-1373.

Muramatsu T, Wang ZC, Fang YR, Hu KB, et al. (1995). Alcohol and aldehyde dehydrogenase genotypes and drinking behavior of Chinese living in Shanghai. Hum. Genet. 96: 151-154.

Nomura T, Noma H, Shibahara T, Yokoyama A, et al. (2000). Aldehyde dehydrogenase 2 and glutathione S-transferase M 1 polymorphisms in relation to the risk for oral cancer in Japanese drinkers. Oral Oncol. 36: 42-46.

Ohsawa I, Kamino K, Nagasaka K, Ando F, et al. (2003). Genetic deficiency of a mitochondrial aldehyde dehydrogenase increases serum lipid peroxides in community-dwelling females. J. Hum. Genet. 48: 404-409. 
Ping Z (2012). Genetic polymorphisms of alcohol dehydrogenase and aldehyde dehydrogenase are associated with coronary artery disease in Chaoshan Han Chinese. Master's thesis, University of Shantou Medical College, Guangdong.

Takagi S, Iwai N, Yamauchi R, Kojima S, et al. (2002). Aldehyde dehydrogenase 2 gene is a risk factor for myocardial infarction in Japanese men. Hypertens. Res. 25: 677-681.

Thakkinstian A, McEvoy M, Minelli C, Gibson P, et al. (2005). Systematic review and meta-analysis of the association between \{beta\}2-adrenoceptor polymorphisms and asthma: A HuGE review. Am. J. Epidemiol. 162: 201-211.

Thomasson HR, Edenberg HJ, Crabb CW, Mai XL, et al. (1991). Alcohol and aldehyde dehydrogenase genotypes and alcoholism in Chinese men. Am. J. Hum. Genet. 48: 677-681.

Wada M, Daimon M, Emi M, Iijima H, et al. (2008). Genetic association between aldehyde dehydrogenase 2 (ALDH2) variation and high-density lipoprotein cholesterol (HDL-C) among non-drinkers in two large population samples in Japan. J. Atheroscler. Thromb. 15: 179-184.

Wang RS, Nakajima T, Kawamoto T and Honma T (2002). Effects of aldehyde dehydrogenase-2 genetic polymorphisms on metabolism of structurally different aldehydes in human liver. Drug Metab. Dispos. 30: 69-73.

Xu F, Chen YG, Geng YJ, Zhang H, et al. (2007). The polymorphism in acetaldehyde dehydrogenase 2 gene, causing a substitution of Glu > Lys(504), is not associated with coronary atherosclerosis severity in Han Chinese. Tohoku J. Exp. Med. 213: 215-220.

Xu F, Chen Y, Lv R, Zhang H, et al. (2010). ALDH2 genetic polymorphism and the risk of type II diabetes mellitus in CAD patients. Hypertens. Res. 33: 49-55.

Xu F, Chen YG, Xue L, Li RJ, et al. (2011). Role of aldehyde dehydrogenase 2 Glu504Lys polymorphism in acute coronary syndrome. J. Cell. Mol. Med. 15: 1955-1962.

Xue L, Chen YG, Xue F, Zhang HE, et al. (2007). Correlation between Aldehyde dehydrogenase-2 gene polymorphisms and myocardial infarction in Chinese Han patients with coronary heart disease. J. Shangdong Univ. 45: 808-812.

Yamada Y, Sun F, Tsuritani I and Honda R (2002). Genetic differences in ethanol metabolizing enzymes and blood pressure in Japanese alcohol consumers. J. Hum. Hypertens. 16: 479-486.

Yijie G (2010). Association of ALDH2 rs671 polymorphism with coronary artery disease susceptibility through affecting endothelial ADMA levels in a Chinese population. ZhongNan University, Changsha, Hunan.

Yokoyama T, Yokoyama A, Kato H, Tsujinaka T, et al. (2003). Alcohol flushing, alcohol and aldehyde dehydrogenase genotypes, and risk for esophageal squamous cell carcinoma in Japanese men. Cancer Epidemiol. Biomarkers Prev. 12: $1227-1233$.

Yuan B (2009). Correlation research between ALDH2 polymorphisms and acute myocardial infarction including inflammation levels of the early phase in Chinese Han people. Master's thesis, Shandong University, Shandong.

Yuan B (2010). Correlation between aldehyde dehydrogenase-2 gene polymorphisms and myocardial infarction in Shandong Han population. J. Aging 30: 2263-2265.

Yuguo CIC (2010). ALDH2 genetic polymorphism and the risk of older of CHD patients. J. Aging 30: 1782-1784. 